\title{
Is it permissible to edit medical records?
}

\author{
Andrew Markus, Michael Lockwood
}

Whatever, in connection with my professional practice, or not in connection with it, I see or hear, in the life of men, which ought not to be spoken of abroad, I will not divulge, as reckoning that all such should be kept secret.

Thus the Hippocratic Oath - by which members of the medical profession, at least in the West, still consider themselves bound-states the principle of confidentiality. As it stands, however, the Hippocratic statement is essentially empty. It binds doctors not to divulge what "ought not to be spoken of abroad" on the strength of the tautology that what ought not to be spoken of abroad "should be kept secret." But it offers no guidance as to what sort of information a doctor is or is not obliged to keep secret, under what circumstances, and from whom. Nor does it tell us who is entitled to decide, in any specific case, whether or not a given item of information can be divulged to a third party, including another doctor or health care worker, and in particular (and of special relevance to the topic of this paper) when the patient should be given power of veto over any such disclosure. The General Medical Council has listed a number of exceptions to the rule of confidentiality, mainly situations in which doctors are permitted to break the rule without the patient's consent.

We shall be better placed to consider the issue of whether it is acceptable to edit medical records when we have first briefly explored the moral basis of confidentiality in medicine.

Two distinct values seem to be at the heart of the principle of confidentiality, one specific to the medical context and the other not. The value not specific to medicine is that of respect for people's privacy. It is generally accepted that there are certain aspects of people's lives which are their own business, and not the business of others (save, perhaps, for a few to whom they have close personal or professional ties). The ability to choose (within certain limits) what others know about us - especially in regard to more intimate personal details - is of importance to us all because it is one of the things that enables us to maintain control over our own lives, and in particular over our relationships with others. That is not to deny that in a close relationship one person may need to know many personal details of the other for trust to be established. Privacy is thus a value closely related to, and perhaps ultimately grounded on, the value of personal autonomy. To take this value of privacy seriously is to subscribe to a number of familiar precepts. It means that we should be reluctant to pry, that we should respect personal confidences, and that when we enter into relationships with others that render us privy to sensitive or intimate personal information we should be chary about passing this information on, even in the absence of any specific request not to do so. (A promise to keep some personal matter secret often serves merely to reinforce an independently existing obligation.) Here, then, is a general principle that applies to everyone-but with particular force to doctors: firstly, because the doctor is especially likely to become aware of sensitive information and, secondly, because the doctor-patient relationship is, par excellence, one based upon trust. These considerations alone would constitute grounds enough for placing doctors under a specific obligation of confidentiality in their professional dealings.

That, however, is only half the story. The other value underlying the principle of confidentiality in medicine is that of the furthering of effective care. A doctor's ability to help a patient is likely to be directly related to the amount the doctor knows about the patient. So the more a patient can rely upon the doctor to treat personal disclosures as confidential, the more open that patient is likely to be, and the better able the doctor may be to provide effective treatment. By the same token, a patient is more likely to consult the doctor in the first place-and thereby receive effective treatment-if he or she can rely on the doctor's discretion in disclosing to third parties sensitive information that emerges in the consultation. These considerations constitute the second main reason for imposing on doctors a specific duty of confidentiality.

Many of the practical difficulties surrounding medical confidentiality in the modern world have to do with the fact that today's doctor is no longer the independent agent that the Hippocratic corpus presumably had in mind. The general practitioner of the $1990 \mathrm{~s}$ is often a member of a group practice, and his or her patients will sometimes need to be referred to colleagues or admitted to hospital. The patient's records, if he or she is an NHS patient, do not even belong to the patient's family doctor, or indeed to any doctor. They are government property, under the ultimate control of the secretary of state. Some 40 people other than the doctors, all with good reason, have access to records in the health centre where one of us (AM) works-reception staff, nurses, health visitors, social workers, clinical psychologists, visiting consultants, medical students, and sometimes others involved in "bona fide" research.

This brings us to the central problem that we are here concerned to address. It seems to us that patients generally give information to their doctors on the understanding that this is to enable that specific doctor to treat them at that particular time. One is not entitled to assume that patients would wish such knowledge to be freely available to any other doctor who might treat them in the future (and perhaps much less to social workers, health visitors, receptionists, medical researchers, and the like). Once such information has been entered into a patient's records, however (quite apart from its becoming immediately accessible, as we have seen, to a number of different people), it will automatically be passed on, by way of the family health services authority, to the patient's next general practitioner, should the patient change doctors.

There can be no doubt that this is sometimes contrary to the patient's own wishes. A case known to one of us (AM) concerned a 21 year old university 


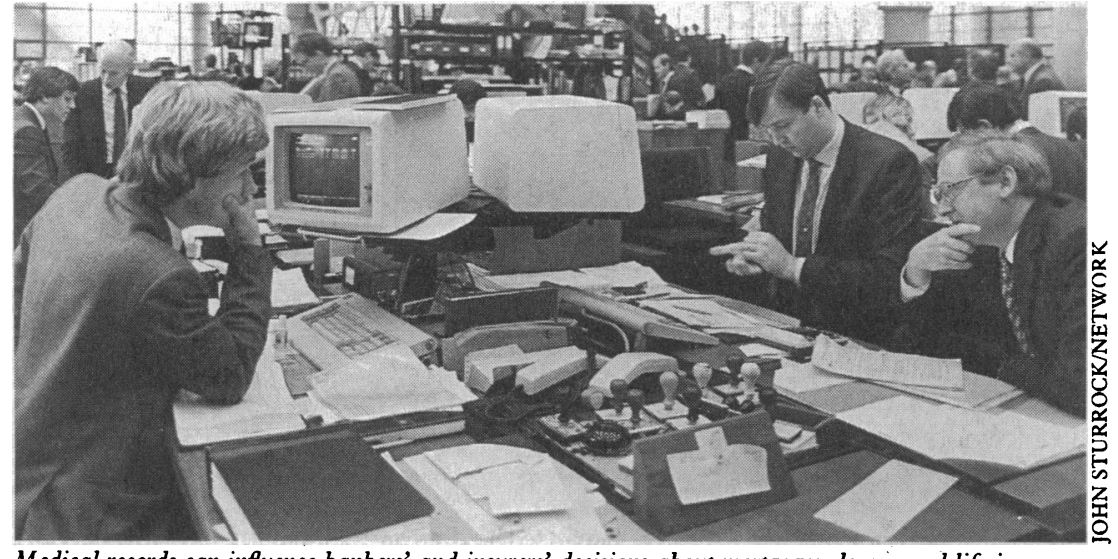

Medical records can influence bankers' and insurers' decisions about mortgages, loans, and life insurance policies. Should patients have a say in what stays on record about them?

student who had suffered a psychotic breakdown during her Oxford career but had nevertheless successfully completed her course. She came to see her doctor after getting her degree to say goodbye and to ask that all mention of the episode should be removed from her records, including hospital letters. She did not want future doctors to label or prejudge her. Another instance was that of a 27 year old male student who was HIV positive and who asked his doctor (AM) to remove all mention of this fact from his records when he was due to leave college. "I gave you that information," he insisted, "not every Tom, Dick, and Harry." A third, hypothetical case (put to ML by a retired general practitioner), is that of a woman whose husband's "sexual shenanigans" are referred to in this woman's records as a possible explanation of symptoms that her doctor was inclined to regard as psychosomatic. It would be only natural for the woman to be concerned, when she moved, that not only her own but her husband's personal affairs would become known to her new general practitioner. It seems to us that in each of these situations a case may be argued for acceding to the patient's wishes.

There are two principles at stake here. The first is that respect for the patient's privacy and autonomy requires that the doctor's disclosure of personal information be sensitive to the intentions and legitimate expectations of the patient. These intentions and expectations will extend both to who is to receive the information and to what it is to be used for. (Thus, it clearly raises ethical problems when a doctor, in connection with an insurance application, is asked to give information that is possibly detrimental to the patient and that was disclosed by the patient for the sole purpose of assisting treatment or diagnosis.)

The second principle is that the patient should not be deprived of the ability to control his or her new doctor's access to personal information that may adversely affect the patient's relationship with that doctor. This goes back to what we identified at the outset as one key rationale of the value of privacy. That the patient who had suffered a psychotic episode be able to start her relationship with her new doctor with a clean slate is surely an entirely reasonable desire and one that demands respect. (It demands respect even if some principle of reciprocity would suggest that a patient has a certain obligation, other things being equal, to cooperate with the doctor to the extent of providing such information as is likely materially to assist diagnosis or treatment, if and when it is needed.)

The case of the HIV patient is more problematic because the new doctor might be argued to have a legitimate interest in the information, relating to his or her own safety. But on the other hand it is recommended practice nowadays that all doctors should behave on the assumption that their patients may be HIV positive.
If the foregoing line of thought is correct then the present situation is profoundly unsatisfactory, for as things now stand it is considered by the General Medical Council to be a disciplinary offence to remove information from records without "adequate justification"; indeed, it would probably constitute falsification of records. This raises the question of what is adequate justification, but it is doubtful whether the mere fact that the patient wants some item of information removed would be regarded by the GMC as such justification. (Where there is a factual error it is of course perfectly permissible to cross out the offending passage, provided it remains legible and the reason for the emendation is made plain.)

At present the general practitioner has no power to prevent the records being passed on to the new doctor. There are moves afoot to allow patients access to their own records, which if realised will probably deter doctors from writing things in the records to which the patient is particularly liable to take exception (though the plan also raises problems of its own). Although no current proposal would seem really to address the problem that concerns us here, access to records might be a first step towards a system in which a certain amount of periodic editing was permitted, on the basis of negotiation between the doctor and the patient. Some set of guidelines would then be needed as to what sorts of requests from patients for deletions in the records should generally be accepted. A more radical proposal would be to follow the practice adopted in some countries, whereby patients have the right to keep their own records and presumably can exercise their own discretion as to how much information is passed on to any new doctor. (It would always be possible to keep duplicates somewhere else in case of loss, and access to these, except perhaps in emergencies, would be allowed only with the patient's express permission.)

Some will no doubt argue that it is in patients' best interests that their new doctor is not deprived of information contained in their previous records, inasmuch as it would not have been recorded by their previous general practitioner had it not been of relevance to their health care. To that, however, there are three answers. In the first place, much of what is recorded is in fact extremely unlikely to be of any future value. Secondly, even under the present system, no doctor is entitled to assume that any records are complete, or even fully accurate: information may be lacking simply because a doctor has never got around to putting it in the notes, or the patient may not have given all relevant facts. This consideration has a bearing, too, on the GMC's view that deletion of relevant information might constitute falsification of records, as also on the argument that such deletions could put doctors themselves at risk. The real moral here is that a doctor should never assume that what is not stated in the records is not, in fact, the case.

But, thirdly, shouldn't it in any case be for the patient ultimately to decide? After all, it is up to the patient what he or she tells the doctor in the first place. If through exercising this choice the patient deprives the new doctor of information that can be of therapeutic or diagnostic value then that, it might be considered, is the patient's own lookout. This view, that in general autonomy should take precedence over what others think might be best for the patient (beneficence), is widely held. It reflects the status of the patient as an adult capable of weighing up the implications of his or her own decisions. Moreover, we think a case can be made out that the patient, in a moral sense, retains a proprietary interest in such personal information as is revealed to the doctor in the course of a consultation. It remains his or her secret, and it should remain for the patient to have the ultimate say as to what use that 
information is put to and to whom it is revealedalways assuming, of course, that no one else's welfare is at stake here. Even if the records, as physical objects, are government property it does not follow that this is true also of the information that they contain. Personal information disclosed in a consultation should perhaps be seen as effectively "on trust" to the doctor for the period of treatment, or for the period in which that doctor continues to have the person as a patient. (The whole issue of ownership of information is one that would merit more attention from moral philosophers.)

Other arguments of a more pragmatic character will no doubt be advanced in favour of maintaining the status quo. One should be reluctant, it may be said, to advocate any new system that would add to the administrative burden under which hard pressed general practitioners and the NHS generally, currently labour. Some sacrifice of patient autonomy may be justified on the basis that it is a relatively small price to pay in return for the very great benefits of the present system, which allows the use of information collected over a long time to be used whenever required in a consultation. But even in therapeutic terms there could be much to be gained by a system that gave patients greater control over their own records; it might well serve to encourage greater openness, enhanced trust, and a greater spirit of cooperation between doctor and patient. We find it difficult to believe that a system more respectful of confidentiality and of the patient's wishes could not be made to work successfully if there was a sufficient will so to do.

1 General Medical Council. Professional conduct and discipline: fitness to practice. London: GMC, 1987:sections 79-88.

\title{
Escape from collective denial: HIV transmission during surgery
}

\author{
A G Bird, S M Gore, A J Leigh-Brown, D C Carter
}

Surgeons are rightly concerned about the risk of acquiring HIV infection when performing operations, ${ }^{1}$ and the risk to patients from an infected surgeon with HIV has been highlighted by two recent events. The Centers for Disease Control reported that an American dentist with AIDS infected as many as five patients during dental care, ${ }^{2}$ and in Britain an infected gynaecologist agreed responsibly that patients he had operated on should be contacted. In the case of the dentist there was unusual similarity in nucleotide sequences of HIV provirus from the dentist and his five patients and two female patients had no other established risk factors for acquiring HIV. Although HIV was prevalent by the late 1970 s in the US, these are the first reported cases of probable operative transmission from a health care worker.

\section{Transmission of bloodborne viruses during surgery}

Members of a surgical team risk percutaneous injuries that could expose either the operator or patient to bloodborne infection. Such injuries occur in $2 \%$ to $15 \%$ of operative procedures, ${ }^{4-5}$ one third of which result in possible reverse exposure (Tokars et al, AIDS conference 1991, Florence).

Transmission of hepatitis B virus from such incidents

HIV Immunology Unit, Royal Infirmary, Edinburgh EH3 9YW

A G Bird, FRCP, senior lecturer

Medical Research Council

\section{Biostatistics Unit,}

Cambridge CB2 2BW

S M Gore, PHD, senior

statistician

B Division of Biological Sciences, University of Edinburgh EH9 3JN A J Leigh-Brown, PHD, lecturer

Department of Surgery, Royal Infirmary, Edinburgh EH3 9YW

D C Carter, FRCS, professor of clinical surgery

Correspondence to: Dr Bird.

BMf 1991;303:351-2 patient. Transmission of the virus can occur at relatively high frequency. Screening of 247 patients operated on by one gynaecologist infected with hepatitis B virus showed that $22(9 \%)$ had markers of hepatitis B infection; the infected women included almost a quarter of those who had undergone hysterectomy (10/42). ${ }^{6}$ Classifying procedures within each surgical specialty as high, medium, or low risk for viral transmission is therefore sensible. ${ }^{?}$

\section{Risk to health care workers from patients}

The transmission of HIV to health care workers after percutaneous injury is being documented prospectively ${ }^{8}$; infection occurs in about $0.4 \%$ of cases but may be influenced by the size of the inoculum..$^{10}$ Hepatitis B virus poses a considerably greater risk than HIV with seroconversion rates of $6 \%$ to $30 \%$ reported after percutaneous incidents involving patients positive for the e antigen. ${ }^{112}$

\section{Assessment of risk of HIV transmission by health workers}

The risk of HIV transmission from an infected health worker during surgery has not been extensively analysed. Only three retrospective studies have been reported, ${ }^{71314}$ yet in Britain alone we estimate that six dentists and 1.6 surgeons have had AIDS diagnosed. ${ }^{15} 16$

In the largest American investigation, testing for HIV antibodies was offered to 1652 patients contacted after a surgeon developed AIDS; 616 (37\%) accepted testing and only one positive result was found in a known intravenous drug misuser. ${ }^{13}$ In a British study 339 patients operated on by a surgeon subsequently found to be HIV positive were contacted and $76(22 \%)$ were tested, all of whom had negative results. ${ }^{7}$

Despite the reassurance of these reports, similarities in the modes of transmission of hepatitis B virus and HIV and the evidence of transmission during dental surgery suggest that perioperative infection will occur, although it is uncertain how often. HIV infection has a long latency so that cases of AIDS appear a long time after surgery and will be more difficult to relate to a surgical event than acute hepatitis associated with hepatitis B virus. ${ }^{12}$ There are currently no virological markers for the likelihood of transmission from individual health workers infected with HIV. ${ }^{17}$

\section{Epidemiological requirements}

The need for data to assess the risk of HIV infection to health care workers has never been questioned yet the same systematic approach has not been adopted for patients. The Royal College of Surgeons in Edinburgh have asked that surgeons should have the right to test for HIV antibodies in patients at risk of infection; they have not suggested anonymised testing of surgeons to quantify the extent of occupational risk. Following the initial Centers for Disease Control report of transmission during dental surgery a working group of the New York Academy of Medicine issued a statement decrying the "substantial public anxiety created concerning what is in fact minimal risk" but did not recommend any studies to document the level of transmission resulting from surgical or dental procedures. ${ }^{18}$ In April 1991 the Department of Health orchestrated that public health officials contact over 1000 women operated on by an infected gynaeco- 